\title{
Changeover without Slot Loss of the Erasure Nodes on DQDB
}

\author{
Tetsuya Yokotani*, Toshihiro Shikama** \\ *Communication Systems Laboratory, \\ Mitsubishi Electric Corporation \\ 5-1-1, Ofuna, Kamakura, Kanagawa, 247, Japan \\ Tel. +81-467-41-2433, Fax. +81-467-41-1050 \\ E-mailyokotani@tsusho.csl.melco.co.jp
}

\author{
**Planing \& administration Department at headquarters, \\ Mitsubishi Electric Corporation \\ 2-2-3, Marunouchi, Chiyoda, Tokyo, 100, Japan \\ Tel. +81-3-3321-2162, Fax. +81-3-3218-2188 \\ E-mailsikama@hat.hon.melco.co.jp
}

\begin{abstract}
This paper concerns the erasure nodes to release slots in DQDB. If the erasure nodes are introduced in the DQDB network, the capability to transfer slots is improved. However, the transit delay from the source node to the destination node is increased by the storage delay in the erasure nodes. We proposed that the number and the location of the erasure nodes are changed according to traffic condition in our pervious work. However, these changes should not disturb the valid slot streams. This paper proposes the scheme to provide this function. This paper also shows the configuration of the erasure nodes having this function. Moreover, it describes the operation algorithm to provide the changeover of a node state, where activation as well as inactivation of slot erasing function in a node is performed without loss of slots. Finally, it estimates the time of this algorithm execution.
\end{abstract}

Keywords

Metropolitan Area Networks, Local Area Networks, Slot reuse, Dual Buses, DQDB 


\section{INTRODUCTION}

DQDB (Distributed Queue Dual Bus) attracts much attention for media access control for LAN as well as MAN. This protocol consists of the physical layer and DQDB layer, and their detailed specifications are described in [IEEE, 1990]. This protocol specifies the fixed length slots for a transmission unit as in Figure 1. The physical layer delineates the slots which are transmitted on some types of transmission frame (e.g., SDH STM-1 [CCITT, 1988]). The DQDB layer specifies the access control in each slot.

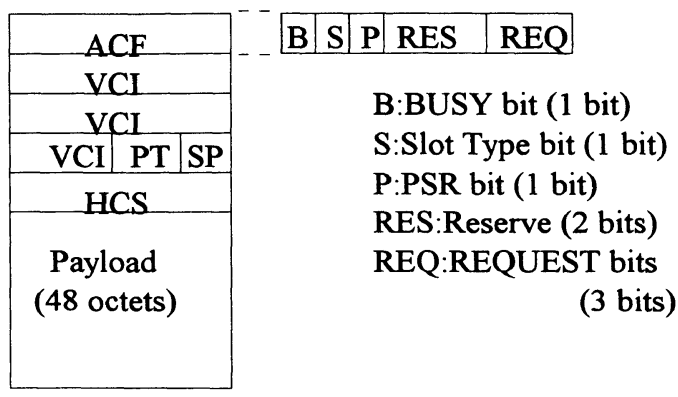

Figure 1 DQDB Slot Format

There are many researches concerning this protocol. One of these items is the slot reusing problem by erasure nodes. The erasure nodes release the fulfilled slots that had finished information transfer. Since these slots can be reused in down stream nodes, the high performance is obtained. Many papers have discussed this issue (e.g., [Breault, 1990], [Perdikaris, 1988], [Rodrigues, 1990] and [Garrett, 1991]). Authors have also proposed the operational mechanisms in the erasure nodes, their configuration and have evaluated the performance ([Yokotani, 1990a], [Yokotani, 1990b], [Yokotani, 1991], [Yokotani, 1992a], [Yokotani, 1992b] and [Yokotani, 1993]). However, while the reuse of slots leads to the enhancement of the throughput, it causes the increase in transit delay from a source node to a destination node because the slots are stored in the erasure nodes. Hence, in the low traffic condition, the erasure nodes degrade the effect on the total performance. Therefore, it is suitable that several nodes have the capability for erasure nodes and the location of the erasure nodes is assigned dynamically according to traffic condition. Authors have pointed out this problem and proposed the control algorithm [Yokotani, 1992a]. However, it should be noticed that the valid streams of slots should not be disturbed in the transition state.

This paper proposes the scheme and its operations for the changeover without loss of slots when the number and the location of the erasure nodes are updated. Moreover, it evaluates the needed period for the completion of these operations.

The structure of this paper is as follows. In Chapter 2, the erasure nodes control 
scheme being proposed by our previous works is summarized. In Chapter 3 , the smoothing switch scheme and the implementation by hardware are described. In Chapter 4 , the needed period for the operation is evaluated.

\section{SUMMARY OF THE CONTROL SCHEME IN THE ERASURE NODES}

This chapter summarizes the control scheme of the erasure nodes according to the traffic condition. The detail of this scheme was described in [Yokotani, 1992a].

\subsection{Configuration}

It is defined that the erasure nodes have two functions. One is the reset of PSR bit (Previous Segment Receive bit) and the other is the cancellation of REQ (REQUEST bit) corresponding to it. Therefore, each node with the slot erasing capability is configured as in Figure 2. When a node becomes an erasure node, the switches are set "On" and the erasure modules are enabled. In this case, the storage delay is added at each erasure module. These operations for BUS A and BUS B are synchronized.

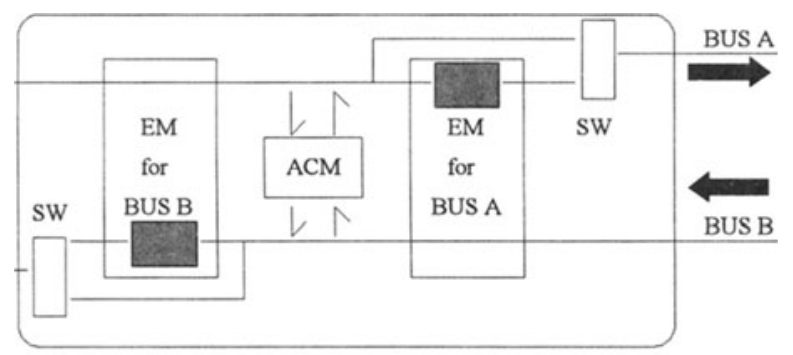

EM:Erasure Module ACM:Access Control Module

SW:Switch to select EM

Figure 2 A Node Configuration

\subsection{Control operations}

The changeover of the erasure nodes is performed as follows.

As in Figure 3, slot terminators with CB (Control Block) control selection of the erasure nodes. This block includes the database with regard to the location of the erasure nodes.

This block monitors each bus and updates the database according to the flow chart as shown in Figure 4. It also calculates the utilization rate $u$, of request slots. Control 
parameters, $a$ and $b(0<b<a<1)$ are introduced. These parameters means the threshold values to control the number and the location of erasure nodes. If this rate is outside the range defined by threshold values $a$ and $b$, the control slot is transmitted from the slot generator on the opposite bus to modify the location and the number of the erasure nodes. As in Figure4, one erasure node is added if $u>a$, and one erasure node is deleted if $u<b$. These operations are invoked for only one node at the same time in each bus. The node that detects the control slot changes its state, the active state or the inactive state of its erasure module. It is executed on both buses at the same time. The detailed operations in these cases are described in [Yokotani, 1992a].

The databases on each bus have to be consistent. Therefore a database must be updated when the control slot is generated on the opposite bus by the above-mentioned algorithm. In other words, as in Figure 4, the database is updated when this function block detects the control slots being transmitted by the slot generator on the same bus, or detects the execution of the above-mentioned algorithm.

These operations provide the modification of the location or the number of the erasure nodes depending on traffic condition.

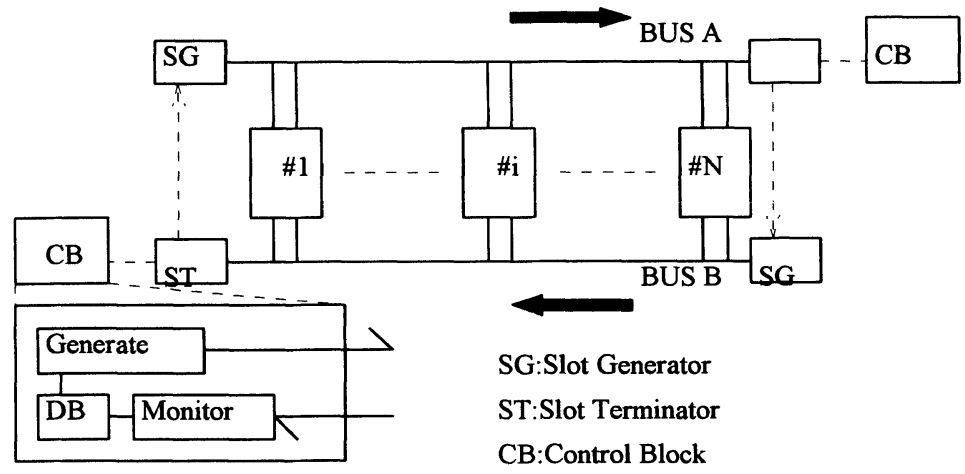

Figure 3 Network Configuration

\section{THE SMOOTH CHANGEOVER SCHEME AND ITS IMPLEMENTATION}

This chapter describes the operation and implementation scheme by hardware to provide the changeover without slot loss of the erasure nodes. 


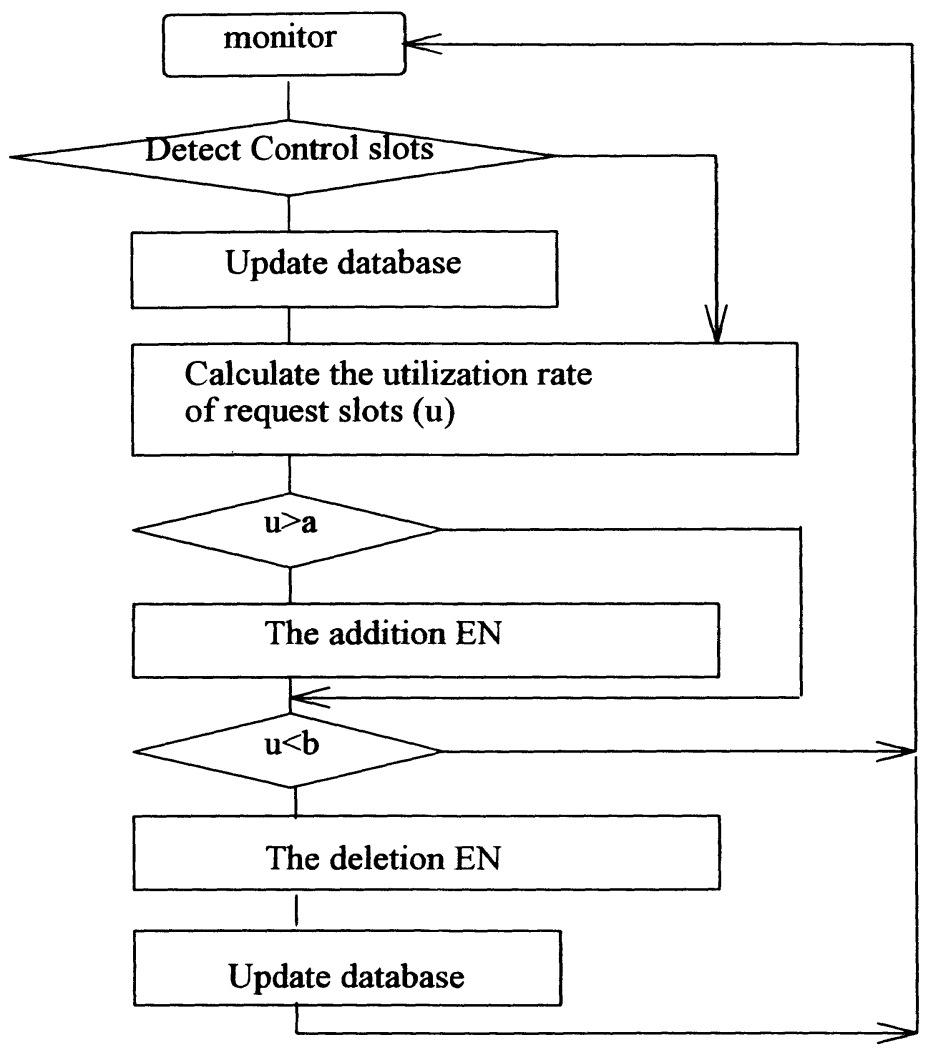

Figure 4 Flow Chart to Update Data Base

\subsection{Physical layer operations}

All receiving slots are sequentially numbered in the physical layer after the delineation of slots. The range of this sequence number is from 0 to 255 . The field for the number is assigned in the second octet of the extended field of slots header as in Figure 5. Because the header of transmission frame is removed, the extended two octets field is assigned available by removing overhead of transmission frame. For example, in SDH STM-1, the transmission rate is $155.52 \mathrm{MHz}$ and the bit rate for transmitting slots excluding SDH frame overhead is $149.76 \mathrm{MHz}$. The bit rate of slots including the extended field is $155.41 \mathrm{MHz}$. It is lower than transmission rate and higher than the bit rate for transmitting slots excluding SDH frame overhead. Moreover, the remaining bandwidth is used by unassigned slots that should not be accessed at DQDB layer and are filled with all 
$1 \mathrm{~s}$ in the first octet of the extended field.

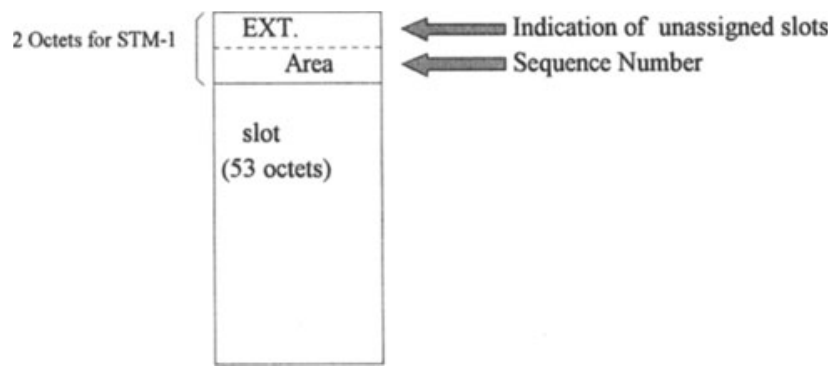

Figure 5 Slot Format with Extended Area for STM-1

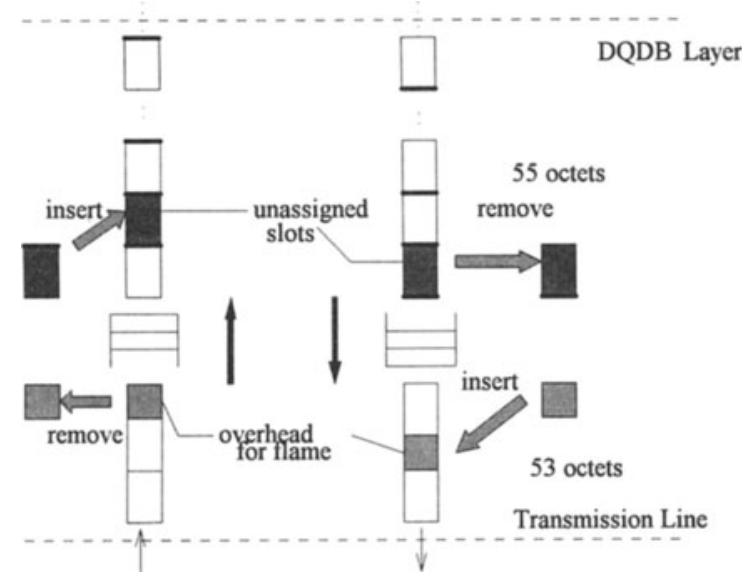

Figure 6 The Processing Slots in Physical Layer

In the physical layer, when slots being received from transmission line are delineated, unassigned slots are periodically stuffed into the remaining bandwidth as in Figure 6.

For example, in SDH STM-1, an unassigned slot is approximately inserted at every 1410 slot. On the other hand, in the operation for transmission, the slots except unassigned slots are mapped into the transmission frame.

Although this paper deals only with the case of the transmission frame of SDH 
STM-1, this scheme can be adopted to other transmission frame specified in [IEEE, 1990] as follows. Because the number of unassigned slots insertion is minimized, the length of the extended area of slot header is extended. The extended area more than two octets is assigned as the bit rate of slots including this area dose not exceed the transmission rate. For example, in case of transmission based on DS3, the extended area must be assigned to five octets including three octets reserved area for minimum insertion of unassigned slots. Thus, an unassigned slot is approximately inserted at every 690 slot.

The detailed operations in case of transmission based on STM-1 are described in the next section.

\subsection{DQDB layer operations}

\subsubsection{The new assigned bit in ACF}

To execute the smooth changeover concerned with an erasure node, a new bit is assigned in ACF as in Figure 8. This bit is called the SV_REQ (Supervisor request bit). The usage of this bit at erasure nodes is described in the following subsection (See 3.3.2 (b)).

Whenever each node detects this bit being set, it performs the same operation as it receives the highest priority request bit.

\subsubsection{Switching operations and implementation scheme}

As in Figure 2, at the switch, the same slots arrive from two paths. However, each arrival time has time lag because the slots outputted from an erasure module is delayed. To change without slot loss, the time lag should be adjusted. The following operations provide such function.

The switches shown as in Figure 2 are configured as in Figure 7. The switching operations at this block are described as follows.

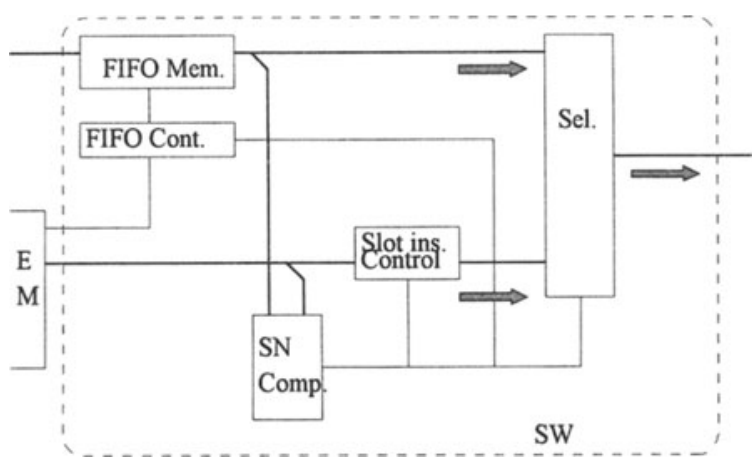

Figure 7 Switch Configuration 


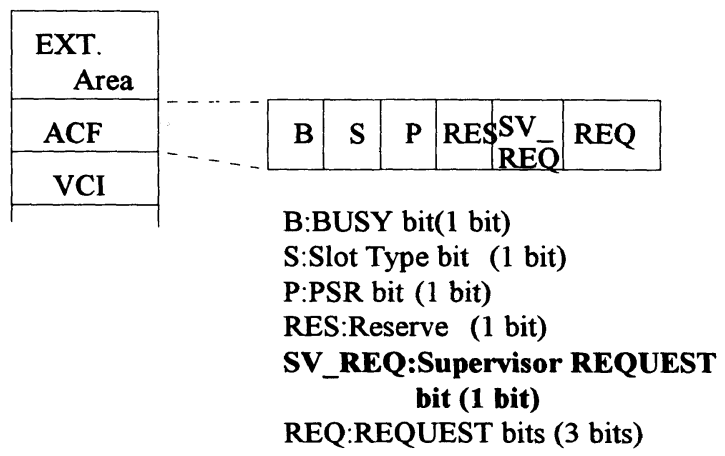

Figure 8 New Assigned Bit in ACF

(a) Erasure Module ON

When the inactive state in the erasure module is changed into active state, the following processes are invoked.

Process 1: The state of erasure module is enabled.

The FIFO Memory with the same capacity as the stored slots in an erasure module is set the active state. All slots including unassigned slots are stored in it.

Process 2: The sequence number of the head slot in the FIFO and that of the outputted slots from an erasure module are compared. If these numbers are identical, the output from an erasure module is selected after this. Otherwise, an unassigned slot is inserted.

Process 3: After the output from an erasure module is selected, the FIFO is reset. Then, these processes are finished.

Comments: the unassigned slots are inserted in Process 2 and these slots are removed in the physical layer. However, the occupied rate of the unassigned slots may exceed the expected rate to execute this process. In this case, the queue in the physical layer to store slots transmitted from DQDB layer to the transmission line may be exhausted. Therefore, in this case, the unassigned slots should be changed into free slots which are QA (Queue Arbitrate) slots without BUSY and REQUEST.

\section{(b) Erasure Module OFF}

When the active state in the erasure module is changed into the inactive state, the following processes are generated. 
Process 1: The FIFO Memory is set active. All slots excepting idle slots (neither BUSY nor REQ) are stored in it. Then, as some idle slots are discarded, the SV_REQs as same as the number of discarding idle slots are generated into the opposite bus.

Because these switching operations are generated for one erasure node at the time in each bus, the slot to set SV_REQ are captured at real time.

Process 2: The sequence number of the head slot in FIFO and of the outputted slots from an erasure module are compared.

If these numbers are identical, the output from a FIFO is selected after this and the state of the erasure module is changed into disable. Otherwise, the output from the erasure module is selected.

Process 3: Until FIFO becomes empty, these processes are executed. Then, these processes are finished.

Comments: Even if the standard ACF of slot header and SV_REQ is not assigned, these operations can be performed. In this case, the highest priority request is set instead of setting SV_REQ. However, if this request bit is already preset by down nodes for normal operation specified in [IEEE, 1990], these operations are suspended until the slot that the highest request bit is not set is captured.

The above-mentioned operations provide the smooth changeover without the disturbance of communications based on the queue arbitrated access protocol in DQDB.

\section{THE EVALUATION OF REQUIRED TIME FOR SWITCHING}

This chapter evaluates the needed period to recover the stable equilibrium after each operation. The common parameters are defined as follows.

$N$ : the delay at an erasure node

$p$ : the rate of the busy slots

In addition to the above definitions, the inter-arrival time of the busy slots which are PA slots or QA slots with BUSY and/or REQUEST follows the geometric distribution with average $1 / p$.

Moreover, the occupied rate of the unassigned slots in total transmission rate is ignored because it is very small. For example, in SDH STM-1, it is only 0.07 percent.

\subsection{The addition of an erasure node case}

In the case of the inactive state being changed into the active state, the needed delay to change state at the erasure node, $A$, is a constant. When $N$ slots period elapses after the switching is required, the first slot after switching is outputted. Then, the node begins to work by an erasure node. 


$$
A=N
$$

\subsection{The deletion of an erasure node case}

In this case of the active state being changed into the inactive state, the process consists of two parts, the stored in the FIFO and the outputted from the FIFO.

\section{(a) The stored in FIFO process}

The time lag between the output from the erasure module and the output from the bypass is $N$ slots time. Therefore, the FIFO stores $N$ slots or less. The probability that $i$ slots are stored in FIFO within $N$ slots time is defined as $P s(i)$.

$$
P_{s}(i)=\left(\begin{array}{c}
N \\
i
\end{array}\right) p^{i}(1-p)^{N-i}
$$

\section{(b) The output from the FIFO process}

It is assumed that $k$ slots are stored in the FIFO within $N$ slots time. After then, the stored slots in the FIFO are outputted at every slot time. However, the new slots arrive at the FIFO according to the arrival rate $p$.

When $k$ slots are stored in the FIFO, the probability that the needed period until the FIFO is empty is $l, P o(k, l)$ is represented as follows.

$$
\begin{aligned}
P_{o}(k, l) & =\left(\begin{array}{c}
l-1 \\
l-k
\end{array}\right) p^{l-k}(1-p)^{(l-l)-(-k)}(l-p) \\
& =\left(\begin{array}{l}
l-1 \\
l-k
\end{array}\right) p^{l-k}(I-p)^{k}
\end{aligned}
$$

From (2) and (3), the average period until the FIFO is empty, $W$, is derived as in (4).

$$
W=\sum_{k=1}^{N} P_{s}(k) \sum_{i=k}^{\infty} l P_{o}(k, l)
$$

Therefore, in this case, the needed period is derived as in (5).

$D=W+N$

The result of the numerical solutions is shown as in Figure 9. 


\subsection{Numerical results and considerations}

The needed delay to change the active state in an erasure node into the inactive state is shown as Figure 9. However, the needed delay to change the inactive state into the active state is constant, or equals to the storage delay in an erasure node $N$. The case that changes the active state into the inactive state is explained as follows.

The average delay to change state of an erasure node depends on the rate of busy slots, $p$, and the storage delay, $N$, in an erasure node. In particular, as the storage delay increases, this characteristic of delay is affected. In heavy traffic case, it shows the outstanding characteristics.

On the other hand, if the storage delay is small, this delay is very small even if traffic is heavy. For example, if $N=1$, it is less than 20 slots time, and if $N=3$, it is less than 60 slots. In SDH STM-1 (i.e., transmission rate is $155.52 \mathrm{MHz}$ ) these are approximately $50 \mu$ s and $150 \mu$ s, respectively.

In our previous research concerned with the erasure nodes (e.g., [Yokotani, 1991]), it is known that the effectiveness does not increase so much even if the storage delay in an erasure node is large. Moreover, if the storage delay is small, the changeover operations can be performed in short cycle and the number and the location of erasure nodes can sensitively respond to traffic condition.

As the results, in that case, this smooth changeover scheme works perfectly to the changing state of the erasure nodes without disturbing the communication. Moreover, it provides to maintain small delay for this changeover.

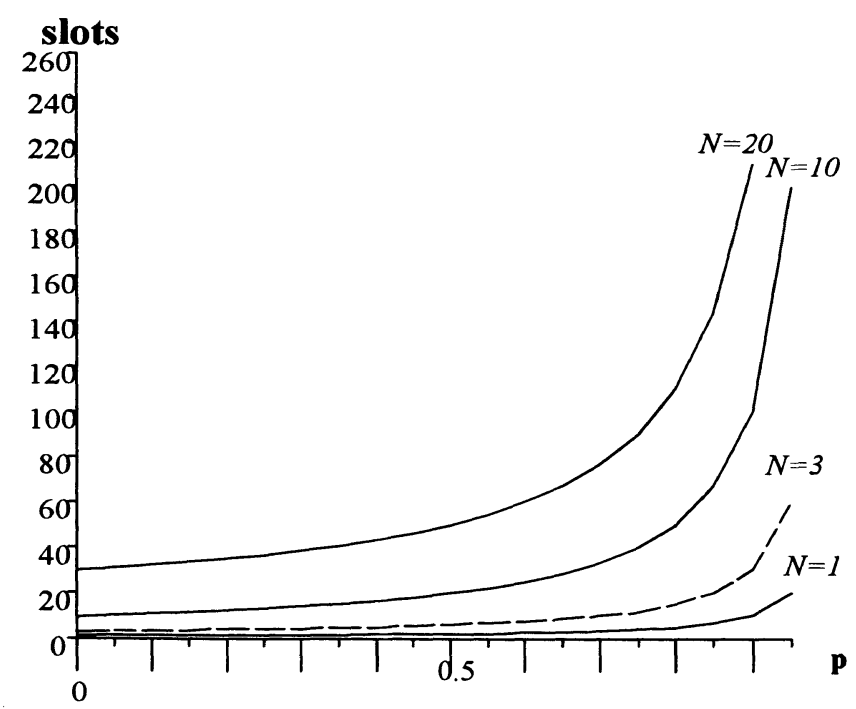

Figure 9 The Average Delay to Change Status of The Erasure Nodes(From the Active State to the Inactive State) 


\section{CONCLUSION}

This paper has dealt with the erasure nodes in DQDB. Though the erasure nodes provide high throughput in the DQDB network, the storage delay that is inevitable in the erasure nodes occupies the large part of total transit delay in the low traffic. Therefore, it is necessary that the number and the location of erasure nodes are changed dynamically. This paper has summarized our previous works on the control scheme for their number and location. Next, we have proposed the scheme to change state of erasure node. Moreover, this paper has shown that the configuration of the hardware to implement its changeover scheme without the disturbing communication. At last, this paper has evaluated the needed period to execute these operations and has shown the characteristic of this delay.

\section{ACKNOWLEDGMENTS}

Authors would like to thank Mr. M. Murotani, Dr. M. Ishizaka and Mr. T. Ichihashi who belong to Mitsubishi Electric Corporation for valuable discussion and comments.

\section{REFERENCES}

[IEEE, 1990] IEEE Standards for Local and Metropolitan Area Networks, "Distributed Queue Dual Bus (DQDB) Subnetwork of a Metropolitan Area Network (MAN)", IEEE Std 802.6

[CCITT, 1988] CCITT Recommendation G.707-G.709, CCITT Blue Books, ITU

[Breault, 1990] R. Breault, V. Phung, "DQDB Erasure Nodes", IEEE contribution 802.690

[Perdikaris, 1988] A.Perdikaris, M.A.Rodrigues, "Destination Release of Segments in the IEEE 802.6 protocol", IEEE contribution 802.6 88/59-61

[Rodrigues, 1990] M.A.Rodrigues, "Performance Improvements for the IEEE 802.6 MAN", INFOCOM'90

[Garrett, 1991] M.W.Garrett, S.Li, "A Study of Slot Reuse in Dual Bus Multiple Access Network", IEEE J.SAC Vol.9 No.2

[Yokotani, 1990a] T.Yokotani, H.Sato, S.Nakatsuka, "Proposed Erasure Nodes Algorithm in DQDB", IEEE 802 Committee Contribution, 802.6 90/49

[Yokotani, 1990b] T.Yokotani, H.Sato, S.Nakatsuka, "Modification of Erasure Nodes Constitution", IEEE 802 Committee Contribution, 802.6 90/83

[Yokotani, 1991] T.Yokotani, H.Sato, S.Nakatsuka, T.Shikama, "The New Slot Reutilization Scheme in DQDB MAN", IEICE Transaction on Communication, E-74 
[Yokotani, 1992a] T.Yokotani, T.Shikama, "The Erasure Nodes Control Scheme on DQDB", GLOBECOM'92, 36A.1

[Yokotani, 1992b] T.Yokotani, T.Shikama, "The Location Control of Erasure nodes in DQDB", Proceedings of the 1992 IEICE Spring Conference, B-434 (in Japanese)

[Yokotani, 1993] T.Yokotani, H.Sato, S.Nakatsuka, "A study on a Performance Improvement Algorithm in DQDB MAN", Computer Networks and ISDN System J. Vol.25, 8

\section{BIOGRAPHIES}

\section{Tetsuya Yokotani:}

He received his B.S. and M.S. degrees from the Science University of Tokyo in 1985 and 1987, respectively. He joined the Mitsubishi Electric Corporation in 1987. Since then he has studied high speed local area networks and ATM networks at the Communication System Laboratory. In particular, he takes interest in the performance evaluation for these systems. $\mathrm{He}$ is a member of the Institute of Electronics, Information and Communication Engineers (IEICE), the Information Processing Academic Society of Japan (IPACJ), the Operations Research Society of Japan (ORSJ), and IEEE.

\section{Toshihiro Shikama:}

He received B.E. and M.E. from Tokyo Institute of Technology in 1974 and 1976, respectively. He joined Mitsubishi Electric Corporation in 1976. Since then he has been engaged in the research and development of a computer network using satellite communications, local area networks, multimedia multiplexers and broadband ISDN. From 1984 to 1985 , he was at the University of Waterloo, where he obtained Master of Science degree. Now, he belongs to planing $\&$ administration department at headquarters. $\mathrm{He}$ is a member of Information Processing Academic Society of Japan (IPASJ). 